\title{
Risk factors for hospital-acquired antimicrobial-resistant infection caused by Acinetobacter baumannii
}

\author{
Darcy Ellis ${ }^{1}$, Bevin Cohen ${ }^{1,2^{*}}$, Jianfang Liu ${ }^{2}$ and Elaine Larson ${ }^{1,2}$
}

\begin{abstract}
Background: Acinetobacter baumannii can cause serious healthcare-associated infections (HAls) and the incidence is increasing, with many strains now resistant to multiple antibiotic classes. The aims of this study were to examine factors associated with HAls caused by antimicrobial-resistant as compared with antimicrobial-susceptible strains of A. baumannii and to investigate trends in the incidence of resistance over time. Electronic data from two U.S. hospitals in a large urban healthcare system in over the years 2006-2012 were used for the analysis. Multiple logistic regression was used to explore risk factors for infection with $A$. baumannii resistant to ampicillin or ampicillin/sulbactam in the bloodstream, urinary tract, and respiratory tract. The Cochran-Armitage trend test was used to explore resistance trends over time.
\end{abstract}

Findings: A total of 671 adults with first-time A. baumannii infection were included in the analysis; 302 isolates (45\%) were resistant to ampicillin or ampicillin/sulbactam and 369 (55\%) were susceptible. In the multivariable analysis, significant risk factors included longer length of stay prior to infection (Odds Ratio $[O R]=1.03 ; 95 \%$ Confidence Interval [Cl]: 1.01, 1.04), hospital A versus B (OR=0.35; $95 \% \mathrm{Cl}: 0.13,0.93)$, and antibiotic use prior to infection ( $\mathrm{OR}=2.88 ; 95 \% \mathrm{Cl}: 1.02,8.13)$. Resistance was more common in respiratory infections $(\mathrm{OR}=2.96 ; 95 \% \mathrm{Cl}$ : $1.04,8.44)$. No trend was found between year of infection and resistance.

Conclusions: The risk factors we identified are consistent with previous findings, but we found no evidence in this population that resistance to ampicillin or ampicillin/sulbactam was increasing over time.

\section{Background}

Acinetobacter baumannii is increasingly implicated as a cause of healthcare-associated infections (HAI), which confer a high risk of morbidity and mortality to patients [1-5]. Infections caused by $A$. baumannii may also be highly resistant to antimicrobials, particularly those strains isolated from critically ill patients in intensive care settings [6-9]. A. baumannii isolates that are resistant to antibiotics can worsen outcomes for patients due to delays in administration of effective therapy, limited treatment options, and high toxicity of available therapies [1]. Risk factors for multidrug-resistant $A$. baumannii colonization and infection include prolonged length of

\footnotetext{
*Correspondence: bac2116@columbia.edu

'Mailman School of Public Health, Columbia, University Medical Center, 722 West 168th Street, New York, NY 10032, USA

${ }^{2}$ School of Nursing, Columbia University Medical Center, 630 West 168th Street, New York, NY 10032, USA
}

hospital stay, exposure to the intensive care unit (ICU), mechanical ventilation, central venous catheterization, urinary catheterization, prior exposure to antimicrobials, greater severity of illness, surgery, and receipt of invasive procedures $[1,10,11]$. Although risk factors for antibioticresistant $A$. baumannii infection have been explored in many patient populations, fewer studies have assessed potential differences in risk factors for those infected with antimicrobial-resistant versus susceptible strains.

Antimicrobial resistance among $A$. baumannii appears to be on the rise internationally $[8,12]$, though temporal changes in antimicrobial susceptibility have not been widely described and may vary locally. Furthermore, research suggests seasonal variation in A. baumannii incidence [13], yet research into this association is limited. Therefore, the aims of this study were to examine factors associated with HAIs caused by antimicrobial-resistant as compared with antimicrobial-susceptible strains of $A$.

\section{() Biomed Central}

(C) 2015 Ellis et al. Open Access This article is distributed under the terms of the Creative Commons Attribution 4.0 International License (http://creativecommons.org/licenses/by/4.0/), which permits unrestricted use, distribution, and reproduction in any medium, provided you give appropriate credit to the original author(s) and the source, provide a link to the Creative Commons license, and indicate if changes were made. The Creative Commons Public Domain Dedication waiver (http://creativecommons.org/publicdomain/zero/1.0/) applies to the data made available in this article, unless otherwise stated. 
baumannii, as well as to investigate trends in the incidence of resistance over time.

\section{Materials and methods Study population}

This retrospective cohort study was conducted as part of a federally funded project, "Health Information Technology to Reduce Healthcare-Associated Infection" (National Institute of Nursing Research, National Institutes of Health; R01NR010822)," which established a clinical research database of patients hospitalized within a large urban healthcare system in New York City. This analysis included all adult ( $\geq 18$ years) discharges occurring from 2006 to 2012 in two tertiary/quaternary care hospitals within the system: a 647-bed adult facility and a 914-bed pediatric/adult facility.

\section{Data collection}

The study database was constructed using electronic data from clinical and administrative systems shared between the study institutions [14]. The primary outcome of interest was the first hospital-acquired (i.e., occurring $>2$ days after hospital admission) bloodstream infection (BSI), urinary tract infection (UTI), surgical site infection (SSI), or pneumonia caused by A. baumannii. Infections were identified using a combination of time-stamped microbiology laboratory records and International Classification of Diseases, Ninth Revision, Clinical Modification (ICD-9-CM) procedure and diagnosis codes, based on modified criteria from the Centers for Disease Control and Prevention National Healthcare Safety Network (NHSN) [15]. BSI were defined as positive A. baumannii blood culture with no positive culture at another body site within the previous 14 days. UTI were defined as positive $A$. baumannii urine culture, i.e., $\geq 10^{5}$ colony forming units (CFU) per $\mathrm{mL}$ of urine and no more than one other species of microorganism, or $10^{3}-10^{5} \mathrm{CFU} / \mathrm{mL}$ plus pyuria. SSI were defined as positive $A$. baumannii wound culture within 30 days of an ICD-9-CM-documented NHSN operative procedure. Pneumonia was defined as positive $A$. baumannii respiratory culture plus any ICD-9-CM code for bacterial pneumonia. Resistance to ampicillin or ampicillin/sulbactam was determined for each infection based on antibiogram data, which were stored electronically for all cultures. The year and season of each infection (Winter, January-March; Spring, April-June; Summer, July-September; Fall, OctoberDecember) were also recorded.

Patients' demographic characteristics and medical conditions were collected from electronic sources. The institution's electronic medication administration record was used to determine whether patients received antibiotics or high risk medications (including chemotherapeutic agents, immunosuppressants, and anti-inflammatory drugs) at least $24 \mathrm{~h}$ before infection. Comorbidities (diabetes, renal failure, and malignancies) and the Charlson Comorbidity Index [16] were collected using ICD-9-CM codes. Electronic administrative records were used to determine patients' age, sex, length of hospitalization, ICU admission, prior within-network hospitalization, and admission from a skilled nursing facility.

\section{Statistical analysis}

The frequency of BSI, UTI, SSI, and pneumonia caused by antibiotic sensitive and resistant $A$. baumannii were recorded by year. The Cochran-Armitage test for trend was used to assess whether the proportion of $A$. baumannii infections that were resistant to antibiotics changed over time. To explore risk factors for HAI caused by antimicrobial resistant versus susceptible $A$. baumannii strains, bivariate analyses between infection and possible predictors were conducted using Pearson's $\chi^{2}$ test for independence (categorical variables) or simple logistic regression (continuous variables). Variables examined included age, gender, hospital (A or B), ICU stay prior to infection, Charlson Comorbidity Index, diabetes, renal failure, malignancy, length of hospital stay prior to infection, antibiotic and high-risk medication use prior to infection, season and year (2006-2012), prior stay in a skilled nursing facility, prior in-network hospitalization, and infection site (BSI, UTI, or pneumonia). SSI were excluded from this analysis due to small sample size. Variables with a $\mathrm{p}$-value less than 0.25 in the bivariate analysis were included in a multivariable logistic regression model in a stepwise forward fashion. All analyses were performed using SAS version 9.4 (SAS Institute, Cary, NC).

\section{Results}

A total of 671 adults with first time hospital-acquired $A$. baumannii infection were identified from 2006 through 2012; 302 patients (45\%) had antimicrobial resistant $A$. baumannii infections and 369 (55 \%) had antimicrobial susceptible infections. Differences between patients with antimicrobial resistant versus susceptible $A$. baumannii infections are described in Table 1. In the final multivariable model, the significant predictors of resistance were length of stay prior to infection $(\mathrm{OR}=1.03 ; 95 \% \mathrm{CI}$ : 1.01 , $1.04)$, being at hospital A vs. B (OR $=0.35 ; 95 \%$ CI: 0.13 , $0.93)$, having a respiratory infection versus other infection types $(\mathrm{OR}=2.96 ; 95 \% \mathrm{CI}: 1.04,8.44)$, and antibiotic use prior to infection $(\mathrm{OR}=2.88 ; 95 \% \mathrm{CI}: 1.02,8.13)$.

Of the total number of first time A. baumannii infections over the study period from 2006 to 2012, 43-68 \% were due to strains resistant to antibiotics. Although there were significant differences by year (chi-square test for independence $p=0.003$ ), there was no significant trend of increasing or decreasing incidence of resistance over time (Cochran-Armitage $p=0.73$; Fig. 1). 
Table 1 Factors associated with healthcare-associated infection caused by antimicrobial resistant versus susceptible Acinetobacter baumannii in two New York City hospitals, 2006-2012

\begin{tabular}{|c|c|c|c|}
\hline & \multicolumn{3}{|c|}{$\begin{array}{l}\text { Resistant to ampicillin or ampicillin/ } \\
\text { sulbactam }\end{array}$} \\
\hline & Yes, N (\%) & No, $N(\%)$ & $P$-value* \\
\hline Total & $302(45 \%)$ & 369 (55 \%) & \\
\hline Mean (range) age, in years & $61(19-98)$ & $61(18-97)$ & 0.65 \\
\hline \multicolumn{4}{|l|}{ Sex } \\
\hline Female & $125(41 \%)$ & $160(43 \%)$ & \multirow[t]{2}{*}{0.61} \\
\hline Male & $177(59 \%)$ & 209 (57\%) & \\
\hline \multicolumn{4}{|l|}{ Hospital } \\
\hline A & $217(76 \%)$ & $190(58 \%)$ & \multirow[t]{2}{*}{$<0.0001$} \\
\hline B & $70(24 \%)$ & 139 (42 \%) & \\
\hline \multicolumn{4}{|l|}{ ICU prior to infection } \\
\hline Yes & $219(83 \%)$ & $178(82 \%)$ & \multirow[t]{2}{*}{0.72} \\
\hline No & $44(17 \%)$ & $39(18 \%)$ & \\
\hline $\begin{array}{l}\text { Mean (range) Charlson } \\
\text { Comorbidity Index }\end{array}$ & $5.61(0-17)$ & $5.39(0-16)$ & 0.40 \\
\hline $\begin{array}{l}\text { Mean (range) days of stay } \\
\text { prior to infection }\end{array}$ & $39(3-377)$ & $18(3-193)$ & $<0.0001$ \\
\hline \multicolumn{4}{|l|}{$\begin{array}{l}\text { High risk medication } \\
\text { prior to infection }\end{array}$} \\
\hline Yes & $136(72 \%)$ & $155(77 \%)$ & \multirow[t]{2}{*}{0.24} \\
\hline No & $53(28 \%)$ & $46(23 \%)$ & \\
\hline \multicolumn{4}{|c|}{ Prior stay in skilled nursing facility } \\
\hline Yes & $21(7 \%)$ & $31(8 \%)$ & \multirow[t]{2}{*}{0.49} \\
\hline No & $281(93 \%)$ & $338(92 \%)$ & \\
\hline \multicolumn{4}{|c|}{ Prior in-network hospitalization } \\
\hline Yes & $72(24 \%)$ & $74(20 \%)$ & \multirow[t]{2}{*}{0.24} \\
\hline No & $230(76 \%)$ & $295(80 \%)$ & \\
\hline \multicolumn{4}{|l|}{ Year of infection onset } \\
\hline 2006 & $32(11 \%)$ & 69 (19\%) & \multirow[t]{7}{*}{0.003} \\
\hline 2007 & $67(22 \%)$ & $65(18 \%)$ & \\
\hline 2008 & $64(21 \%)$ & $48(13 \%)$ & \\
\hline 2009 & $40(13 \%)$ & $52(14 \%)$ & \\
\hline 2010 & $36(12 \%)$ & $44(12 \%)$ & \\
\hline 2011 & $41(14 \%)$ & 47 (13\%) & \\
\hline 2012 & $22(7 \%)$ & $44(12 \%)$ & \\
\hline \multicolumn{4}{|l|}{ Site of infection } \\
\hline Bloodstream & $65(22 \%)$ & $85(23 \%)$ & 0.64 \\
\hline Urinary tract & 96 (32 \%) & $123(33 \%)$ & 0.67 \\
\hline Pneumonia & 187 (62 \%) & $172(47 \%)$ & $<0.0001$ \\
\hline \multicolumn{4}{|l|}{ Season of infection onset } \\
\hline Winter & $67(22 \%)$ & $83(22 \%)$ & \multirow[t]{4}{*}{0.75} \\
\hline Spring & $76(25 \%)$ & 95 (26 \%) & \\
\hline Summer & $85(28 \%)$ & $113(31 \%)$ & \\
\hline Fall & 74 (25 \%) & 78 (21 \%) & \\
\hline
\end{tabular}

Table 1 Factors associated with healthcare-associated infection caused by antimicrobial resistant versus susceptible Acinetobacter baumannii in two New York City hospitals, 2006-2012 (Continued)

\begin{tabular}{lccc}
\hline $\begin{array}{l}\text { Diabetes } \\
\text { Yes }\end{array}$ & $100(33 \%)$ & $106(29 \%)$ & 0.22 \\
No & $202(67 \%)$ & $263(71 \%)$ & \\
Renal failure & & & \\
Yes & $181(60 \%)$ & $179(49 \%)$ & 0.003 \\
No & $121(40 \%)$ & $190(51 \%)$ & \\
Malignancy & & & \\
Yes & $69(23 \%)$ & $79(21 \%)$ & 0.65 \\
No & $233(77 \%)$ & $290(79 \%)$ & \\
Antibiotic use prior to infection & & & \\
Yes & $16(40 \%)$ & $20(20 \%)$ & 0.017 \\
No & $24(60 \%)$ & $78(80 \%)$ & \\
\hline
\end{tabular}

Numbers in strata may not equal total due to missing values ${ }^{*}$ Continuous variables assessed using simple logistic regression (Wald $\chi^{2}$ ). Categorical variables assessed using Pearson's $X^{2}$

\section{Discussion}

According to the most recent NHSN report, among $A$. baumannii infections identified in 2009-10, $78 \%$ of catheter-associated urinary tract infections, $67 \%$ of central-line associated bloodstream infections had multidrug resistance, and $99 \%$ of ventilator-associated pneumonia were multidrug resistant [5]. However, other studies have reported rates of multidrug resistance between 51 and $54 \%[17,18]$, which are closer to the rate of resistance found in this study (45\%). Estimates of the prevalence of resistance may vary due to differences in the definitions of antimicrobial resistance used across studies, as well as differences in the types of infections studied, patient case mix, and geographic location. Seasonality, however, does not appear to play a role.

Although rates of resistance differed by year, there was no significant trend over time. A previous report from New York City comparing resistance to ampicillin/sulbactam across three time points found an increase between 1999 and 2001 (34\% to $46 \%$ ), though this trend did not continue into 2006 (44 \%) [19]. Other U.S. studies, however, have reported large increases in resistance over the past decade. A study conducted in Detroit found that between 2003 and 2008, the total number of patients with $A$. baumannii infection increased, as did resistance to most antibiotics, with ampicillin/sulbactam resistance climbing from $11 \%$ to $60 \%$ [20]. NHSN described an increase in multidrug resistance covering six classes including ampicillin/sulbactam from $50 \%$ in $2007-8$ to $63 \%$ in $2009-10$ [5]. It appears, therefore, that there is no consistent trend in ampicillin/sulbactam 


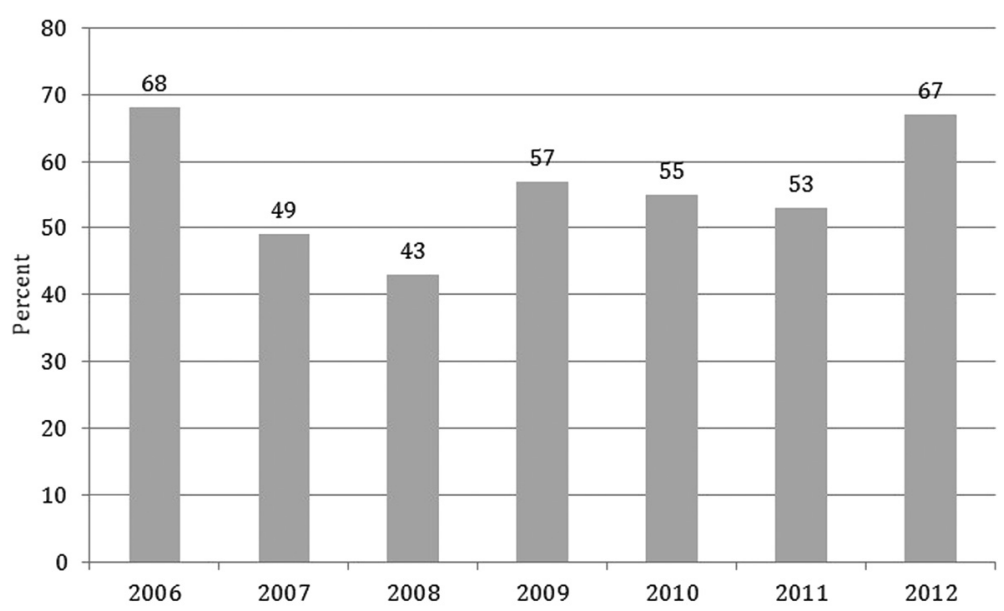

Fig. 1 Percent of hospital-acquired Acinetobacter baumannii strains resistant to ampicillin or ampicillin/sulbactam in two New York City hospitals, 2006-2012. Pearson's $x 2$ test for independence across years, $p=0.003$. Two-sided Cochran-Armitage test for trend, $p=0.73$

resistance for A. baumannii, and that trends may be primarily associated with local practices or other regional differences.

This study identified four risk factors for hospitalacquired $A$. baumannii resistant infection. Consistent with other studies, a longer hospital stay prior to infection, infection in the respiratory tract, and antibiotic use before infection were all significant predictors of antimicrobial resistance $[1,2,12]$. Greater resistance among respiratory tract infections may be explained in part by high levels of A. baumannii contamination on respirators and suctioning equipment, particularly in ICUs, which may lead to environmental reservoirs of resistant strains [21]. This finding highlights the importance of environmental hygiene for preventing A. baumannii infections in the respiratory tract, especially for ventilated patients. Unexpectedly, being in hospital A vs. B was also a significant predictor, controlling for potential confounders such as comorbidities, medications used, other host characteristics, and length of stay. There are no obvious reasons why resistance varied significantly between the two hospitals, given that both are tertiary/quaternary care hospitals within the same hospital system. It is possible that there were other host or environmental factors which were unaccounted for, though this could not be assessed due to the retrospective nature of our study. While the infection prevention policies were the same in both hospitals, it is also possible that infection prevention practices varied $[22,23]$.

There are some limitations to this study. Firstly, we did not examine factors associated with resistance among patients with surgical site infections because the numbers were too few. Secondly, data were limited to what was available from electronic data sources. Although the electronic algorithms used to detect infections are based on NHSN criteria and have been validated by a clinical team, the "gold standard" for identifying infections is clinician diagnosis. In addition, information on the molecular characteristics of $A$. baumannii isolates were not available, so subtyping could not be performed. Furthermore, some patient comorbidities were identified by ICD-9-CM codes, which have been shown to have poor sensitivity for many chronic conditions [24]. Lastly, this study was conducted using data from a single healthcare system in the United States, a region where $A$. baumannii is not endemic. Thus the findings may not be generalizable to other populations.

The results of this study confirm what some other studies have shown, that the length of hospital stay and antibiotic use prior to infection are significantly associated with increased risk of an antimicrobial resistant $A$. baumannii infection, and that resistance was more common in the respiratory tract as compared to other body sites. Unlike other studies investigating risk factors for infection, this study also examined trends in resistance over a seven year period and found no clear pattern in the prevalence of resistance to ampicillin/sulbactam among $A$. baumannii strains.

\section{Ethics, consent and permissions}

This study was approved by the Columbia University Medical Center Institutional Review Board. A waiver of informed consent was granted.

\section{Competing interests}

The authors declare that they have no competing interests.

\section{Authors' contributions}

DE performed the analyses and drafted the manuscript. BC contributed to manuscript writing. JL provided the study data and consulted on the analysis. EL contributed to the conceptualization of the study and manuscript writing. All authors read and approved the final manuscript.

\section{Acknowledgements}

This study was funded by the National Institute for Nursing Research, National Institutes of Health (NR010822). 
Received: 1 July 2015 Accepted: 2 October 2015

Published online: 09 October 2015

\section{References}

1. Maragakis L, Perl T. Acinetobacter baumannii: Epidemiology, Antimicrobial Resistance, and Treatment Options. Clin Infect Dis. 2008;46:1254-63.

2. Ozgur ES, Horasan ES, Karaca K, Ersoz G, Nayci Atis S, Kaya A. Ventilatorassociated pneumonia due to extensive drug-resistant Acinetobacter baumannii: Risk factors, clinical features, and outcomes. Am J Infect Control. 2014;42:206-8

3. Marshall C. A longitudinal study of Acinetobacter in three Australian hospitals. J Hosp Infect. 2007;67:245-52.

4. Hidron Al, Edwards JR, Patel J, Horan TC, Sievert DM, Pollock DA, et al. Antimicrobial-resistant pathogens associated with healthcare-associated infections: annual summary of data reported to the National Healthcare Safety Network at the Centers for Disease Control and Prevention, 2006-2007. Infec Control Hosp Epidemiol. 2008;29:996-1011.

5. Sievert DM, Ricks P, Edwards JR, Schneider A, Patel J, Srinivasan A, et al. Antimicrobial-resistant pathogens associated with healthcare-associated infections: summary of data reported to the National Healthcare Safety Network at the Centers for Disease Control and Prevention, 2009-2010. Infec Control Hosp Epidemiol. 2013;34:1-14.

6. Cisneros J, Rodriguez-Bano J. Nosocomial bacteremia due to Acinetobacter baumannii: Epidemiology, clinical features and treatment. Clin Microbiol Infec. 2002;8:687-93.

7. Giamarellou H, Antoniadou A, Kanellakopoulou K. Acinetobacter baumannii: A universal threat to public health. Int J Microbiol Agents. 2008;32:106-19.

8. Dijkshoorn L, Nemec A, Seifert H. An increasing threat in hospitals: Multidrug-resistant Acinetobacter baumannii. Nature Rev Microbiol. 2007:5:939-51.

9. Fournier PE, Richet $\mathrm{H}$. The epidemiology and control of $A$. baumannii in health care facilities. Healthcare Epidemiology. 2006:42:692-9.

10. Playford E, Craig J, Iredell J. Carbapenem-resistant Acinetobacter baumannii in intensive care unit patients: Risk factors for acquisition, infection and their consequences. J Hosp Infect. 2007;65:204-11.

11. Zhou H, Yuan Z, Du Y. Prior use of four invasive procedures increases the risk of Acinetobacter baumannii nosocomial bacteremia among patients in intensive care units: A systematic review and meta-analysis. Int J Infect Dis. 2014:22:25-30.

12. Villar M, Cano ME, Gato E, Garnacho-Montero J, Miguel Cisneros J, et al. Epidemiologic and Clinical Impact of Acinetobacter baumannii Colonization and Infection. Medicine. 2014;93:202-10.

13. Bergogne-Berezin E, Towner K. Acinetobacter spp. as Nosocomial Pathogens: Microbiological, Clinical, and Epidemiological Features. Clin Microbiol Rev. 1996;9:148-65.

14. Apte M, Neidell M, Furuya EY, Caplan D, Larson E. Using Electronically Available Inpatient Hospital Data for Research. Clin Transl Sci. 2011;4:338-45.

15. Horan TC, Andrus M, Dudeck MA. CDC/NHSN surveillance definition of health care-associated infection and criteria for specific types of infections in the acute care setting. Am J Infect Control. 2008;36:309-32.

16. Charlson M, Pompei P, Ales K, Mackenzie C. A new method of classifying prognostic comorbidity in longitudinal studies: Development and validation. J Chron Dis. 1987:40:373-83.

17. Sunenshine RH, Wright MO, Maragakis LL, Harris AD, Song X, Hebden J, et al. Multidrug-resistant Acinetobacter infection mortality rate and length of hospitalization. Emerg Infect Dis. 2007;13:97-103.

18. Queenan AM, Pillar CM, Deane J, Sahm DF, Lynch AS, Flamm RK, et al. Multidrug resistance among Acinetobacter spp. in the USA and activity profile of key agents: results from CAPITAL Surveillance 2010. Diagn Microbiol Infect Dis. 2012;73:267-70.

19. Landman D, Bratu S, Kochar S, Panwar M, Trehan M, Doymaz M, et al. Evolution of antimicrobial resistance among Pseudomonas aeruginosa, Acinetobacter baumannii, and Klebsiella pneumoniae in Brooklyn. NY J Antimicrob Chemother. 2007;60:78-82.

20. Reddy T, Chopra T, Marchaim D, Pogue JM, Alangaden G, Salimnia H, et al. Trends in antimicrobial resistance of Acinetobacter baumannii isolates from a metropolitan Detroit health system. Antimicrob Agents Chemother. 2010;54:2235-8.

21. Manchanda V, Sanchaita S, Singh NP. Multidrug Resistant Acinetobacter. J Glob Infect Dis. 2010;2:291-304.
22. Karageorgopoulos DE, Falagas ME. Current control and treatment of multidrug-resistant Acinetobacter baumannii infections. Lancet Infect Dis. 2008:8:752-62.

23. Bayuga S, Zeana C, Sahni J, Della-Latta P, El-Sadr W, Larson E. Prevalence and antimicrobial patterns of Acinetobacter baumannii on hands and nares of hospital personnel and patients: The iceberg phenomenon again. Heart Lung. 2002;31:382-90.

24. Quan H, Li B, Saunders LD, Parsons GA, Nilsson Cl, Alibhai A, et al. Assessing validity of ICD-9-CM and ICD-10 administrative data in recording clinical conditions in a unique dually coded database. Health Serv Res. 2008;43:1424-41.

\section{Submit your next manuscript to BioMed Central and take full advantage of:}

- Convenient online submission

- Thorough peer review

- No space constraints or color figure charges

- Immediate publication on acceptance

- Inclusion in PubMed, CAS, Scopus and Google Scholar

- Research which is freely available for redistribution 